\title{
Recurrent episodes of fever and arthritis in an adult patient
}

\author{
Claudia Cobilinschi', Daniela Opris-Belinski ${ }^{1,2}$, Denisa Predeteanu ${ }^{1,2}$, Ruxandra Ionescu ${ }^{1,2}$ \\ ${ }^{1}$ Department of Rheumatology and Internal Medicine, "Sf. Maria" Clinical Hospital, \\ Bucharest, Romania \\ 2"Carol Davila” University of Medicine and Pharmacy, Bucharest, Romania
}

\begin{abstract}
Familial Mediterranean Fever (FMF) is the most common condition in the group of autoinflammatory diseases. It
has a relatively heterogeneous clinical display included in the Tel-Hashomer diagnostic criteria that can be further
confirmed by genetic testing showing a mutation in the MEFV gene. AA Amyloidosis is the most sever complica-
tion in these patients. Colchicine is the standard treatment and/or alternative biological agents if necessary. This
article aims to describe the evolution of a patient with FMF, firstly diagnosed as reactive arthritis, his clinical mani-
festations and therapeutic options.
\end{abstract}
Keywords: Familial Mediterranean Fever, genetic diagnosis, amyloidosis, colchicine

\section{INTRODUCTION}

Familial Mediterranean Fever (FMF) is a relatively rare condition that belongs to the more recent group of autoinflammatory diseases (AIDs) (1). It primarily affects patients with Mediterranean or Middle Eastern origins and its clinical setting includes short, recurrent episodes of fever, serositis, skin rash and a high risk of AA amyloidosis (2), having a significant impact on quality of life even in the attack-free periods (3). FMF is an autosomal recessive transmitted disease and several mutations of the Mediterranean fever gene (MEFV) on chromosome 16 have been identified (4). Patients respond well to colchicine therapy or if necessary, biological therapy with anti-IL 1, anti-IL6 or anti-TNF could be initiated (5). Establishing the right diagnosis might raise difficulties for rheumatologists who are not fully accustomed to this condition.

\section{CASE DESCRIPTION}

This is the case report of a 37-year old male patient recently admitted in our Rheumatology Department for right knee arthritis and high grade fever. Both his lifestyle background and family history are non-significant. Patient's physical examination reveals a relatively impaired general state with pale, dehydrated skin and diffuse stretch marks. He has an important swelling in his right knee with increased local heat and limited hip mobility. His vitals show tachycardia and fever, with a blood pressure value of $120 / 80 \mathrm{mmHg}$, heart rate at $100 \mathrm{bpm}$ and temperature varying between 38.5 and $39.1^{\circ} \mathrm{C}$ with three febrile peaks, while his lab values display a high inflammatory syndrome (high ESR \& CRP) and no leukocytosis. A right knee arthrocentesis was performed resulting in $75 \mathrm{ml}$ of opalescent liquid; cultures from the synovial fluid came back negative.

Patient's medical history dates back at age 16 when he first presented in a Pediatric Ward with recurrent episodes of prolonged fever, of up to $40^{\circ} \mathrm{C}$, diffuse abdominal pain together with myalgia, arthralgia accompanied by increased acute phase reactants (ESR $98 \mathrm{~mm} / \mathrm{h}, \mathrm{CRP} 154 \mathrm{mg} / \mathrm{l})$. After various sources of infection and hematological malignancies (hepatitis, rheumatic fever, leukemia or lymphoma) were excluded, physicians noted positive low titer ANA (1/20) but with normal complement fractions and absent lupus cells. He was left on an intermittent 
regimen of non-steroidal anti-inflammatory drugs (NSAIDs) until the age of 18 when he presents with recurrent arthritis in his right knee accompanied by markedly elevated inflammatory markers (ESR 128 $\mathrm{mm} / \mathrm{h}$, CRP $52.4 \mathrm{mg} / \mathrm{l})$. Physicians undergo repeated punctures of the knee joint and intraarticular glucocorticoids.

Further medical investigations showed a negative rheumatoid factor, negative ACPA, together with a negative extensive antibody panel (dsDNA, Sm, Ro, U1-RNP, anti-phospholipid) and absent cryoglobulins but a positive HLA B27. However, no signs of sacroiliitis were detected neither on the x-ray performed at the time nor on subsequent hip MRIs.

Patients' repeated complaints of knee and ankle arthritis together with later finding of positive antiSalmonella and anti-Shigella antibodies (titer $>1 / 160$ ) led to establishing the diagnosis of reactive arthritis. Due to symptoms' persistence and reoccurrence under NSAIDs, he was prescribed high dose corticosteroids (Methylprednisolone $16 \mathrm{mg}$ bid) and sulfasalazine $2 \mathrm{~g}$ per day. At the age of 20 the patient presents with arthritis in his knees and ankles, a three day ongoing fever of up to $40^{\circ} \mathrm{C}$ and abdominal pain. He exhibits no signs of rash, uveitis or aphtous lesions. His second phase reactants indicate a significant inflammatory syndrome, with a ESR of 115 $\mathrm{mm} / \mathrm{h}$ and a CRP of $154 \mathrm{mg} / \mathrm{l}$, with mild anemia (Hb $11.9 \mathrm{~g} / \mathrm{dl})$.

His procalcitonin level was within normal range and the cardiac ultrasound showed no signs of bacterial endocarditis or pericardial effusion. The abdominal ultrasound highlighted a splenomegaly and peritonitis.

Taking into account the features of the patient's clinical setting, physicians at the time suggested the possibility of Familial Mediterranean Fever as diagnosis, thus colchicine treatment $1 \mathrm{mg}$ per day was promptly initiated. His rapid favorable response led to further genetic testing of the FMF gene (MEFV) in 2002 that revealed a mutation of the 10.1 exon, the patient being homozygous for M694, thus confirming the diagnosis of FMF by fulfilling two major criteria of the Tel-Hashomer diagnostic set.

Subsequently, he performed a gingival biopsy that excluded the presence of amyloid deposits. Patient's partial response to colchicine with repeated incomplete attacks of FMF and a persistently high inflammatory syndrome imposed the initiation of biological therapy with an anti-TNF agent, namely Etanercept, due to the temporary unavailability of an anti-IL1 agent.

In 2015 the patient presents with arthritis in the same knee, as seen in the pictures below.
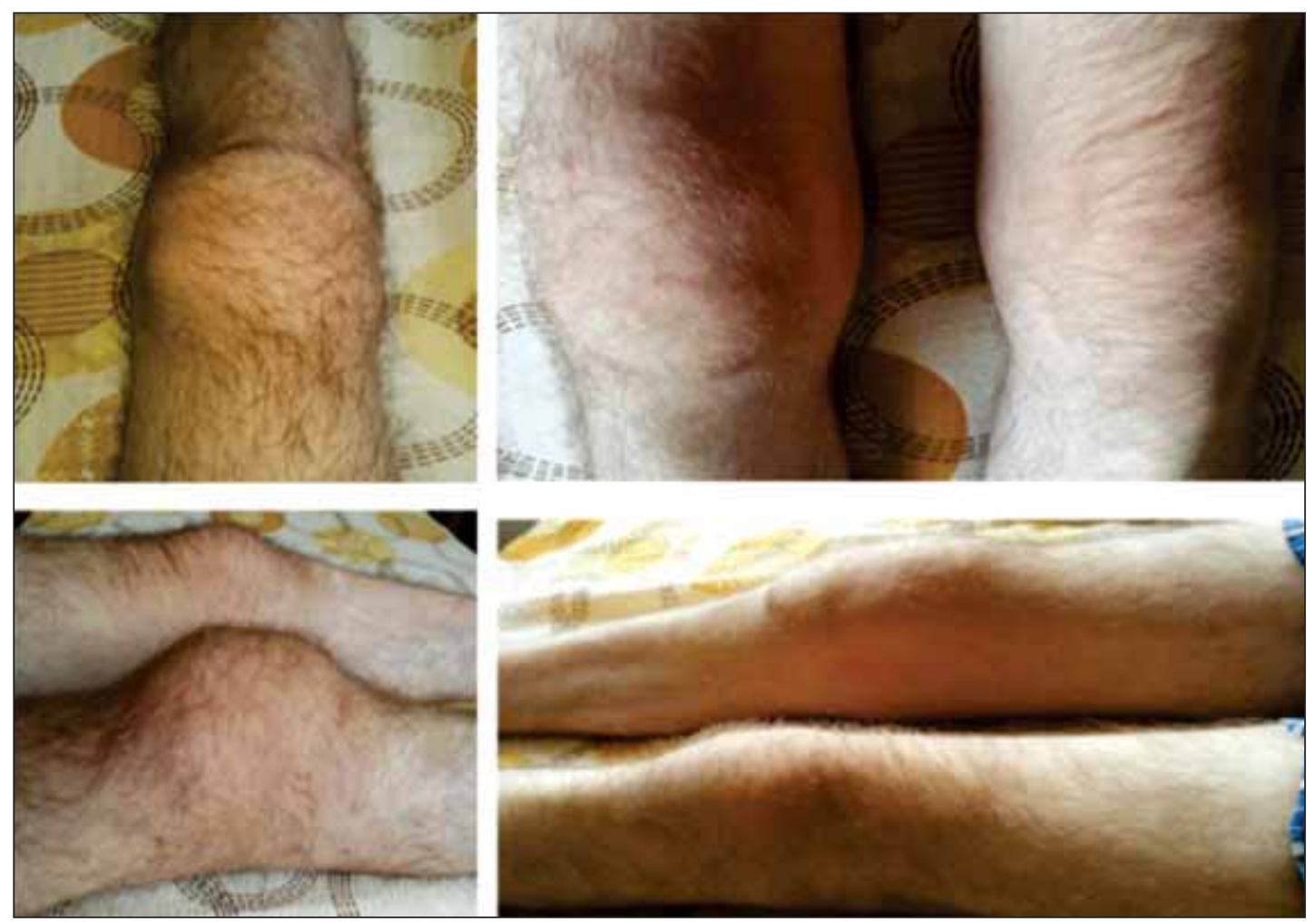

FIGURE 1. Significant swelling of the right knee Source: Author's contribution 


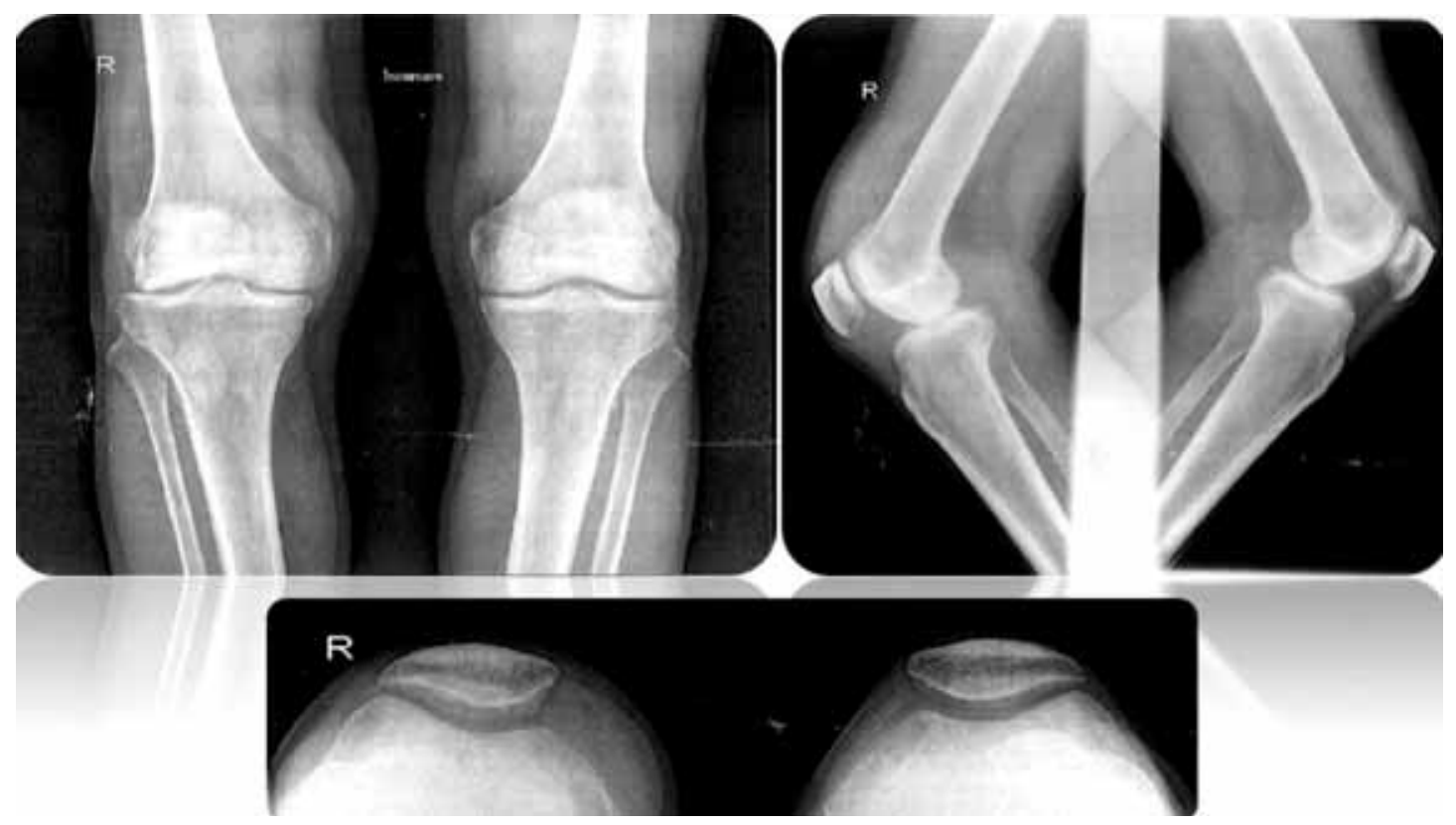

FIGURE 2. Knee x-rays showing severe joint space narrowing

Source: Author's contribution

X-rays were performed showing severe joint space narrowing affecting bilateral compartments.

A partial synovectomy was carried out together with a synovial biopsy showing areas of fibrinoid necrosis, focal reactive mesothelial hyperplasia, frequent capillaries, lympho-plasmocytic inflammatory infiltrate, suggesting a chronic, non-specific synovitis with no signs of granulomatous inflammation at the microscopic examination.

Bone densitometry was performed in 2016 showing severe osteoporosis with a vertebral $\mathrm{T}$ score of -4.2 and a lumbar MRI that confirmed multiple microfractures of the T12 to L5 vertebrae with diffuse demineralization.

Repeated hand imaging through both x-rays and ultrasound showed no involvement of the small joints, with the absence of erosions and no joint space narrowing.

Because of his limited hip mobility assessed at the clinical examination, the patient had both a hip $\mathrm{x}$-ray and MRI taken that certified the presence of bilateral aseptic osteonecrosis of the femoral head, requiring hip arthroplasty due to its severe extent.

He recently suffered from a ruptured Baker's cyst in this left knee, in February 2017 for which the patient had orthopedic excision, local debris removal and area sterilization.

The following pictures depict patient's current state with no hand deformities, with a residual knee arthritis and his difficulties in ambulation that is only made possible with crutches.

Following this extensive case report, we can summarize patient's diagnosis as follows: he suffers from late-onset Familial Mediterranean Fever with a 10.1 exon mutation and a left fused Baker's cyst with recent surgical excision. He also has bilateral aseptic femoral head osteonecrosis, severe osteoporosis with vertebral fractures, bilateral steroid induced cataract and a iatrogenic Cushing's syndrome.

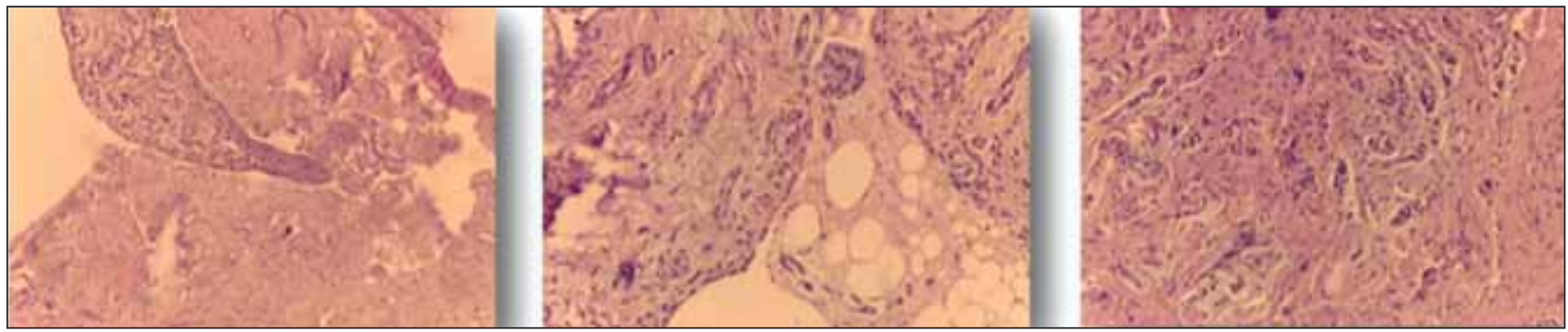

FIGURE 3. Microscopic findings of the synovium Source: Author's contribution 

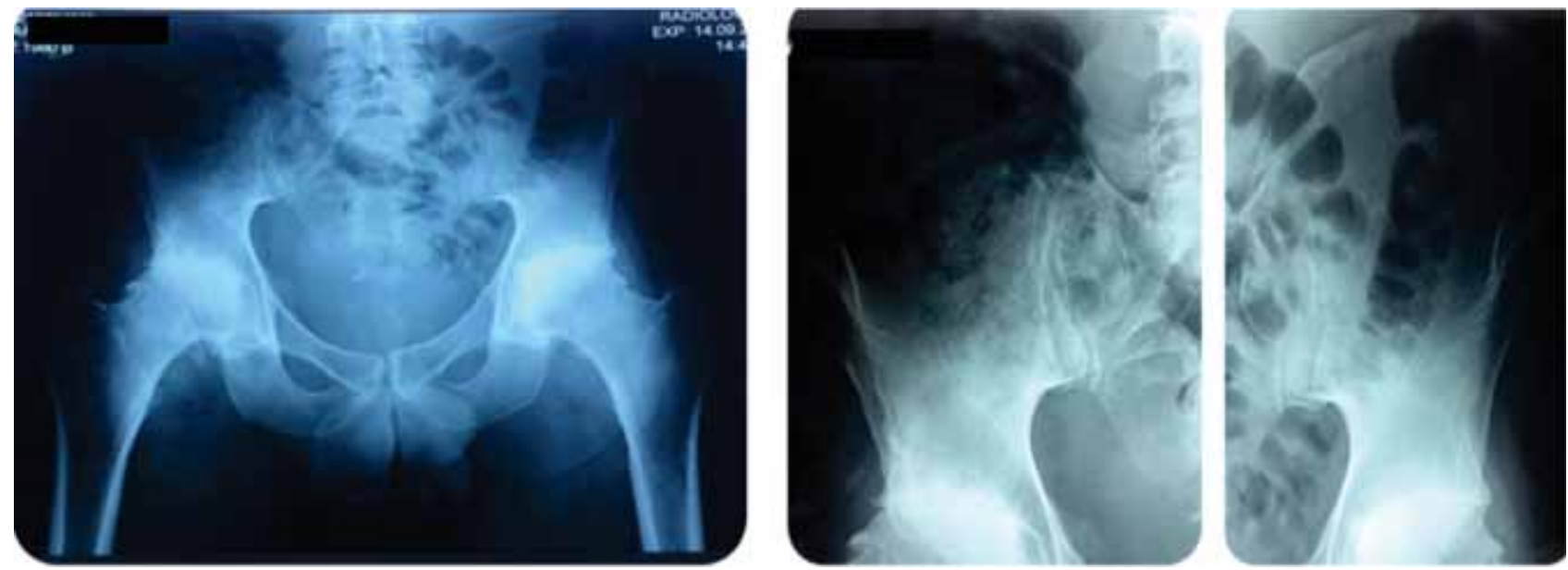

FIGURE 4. Hip x-rays showing severe femoral head osteonecrosis and no sacroiliac involvement Source: Author's contribution

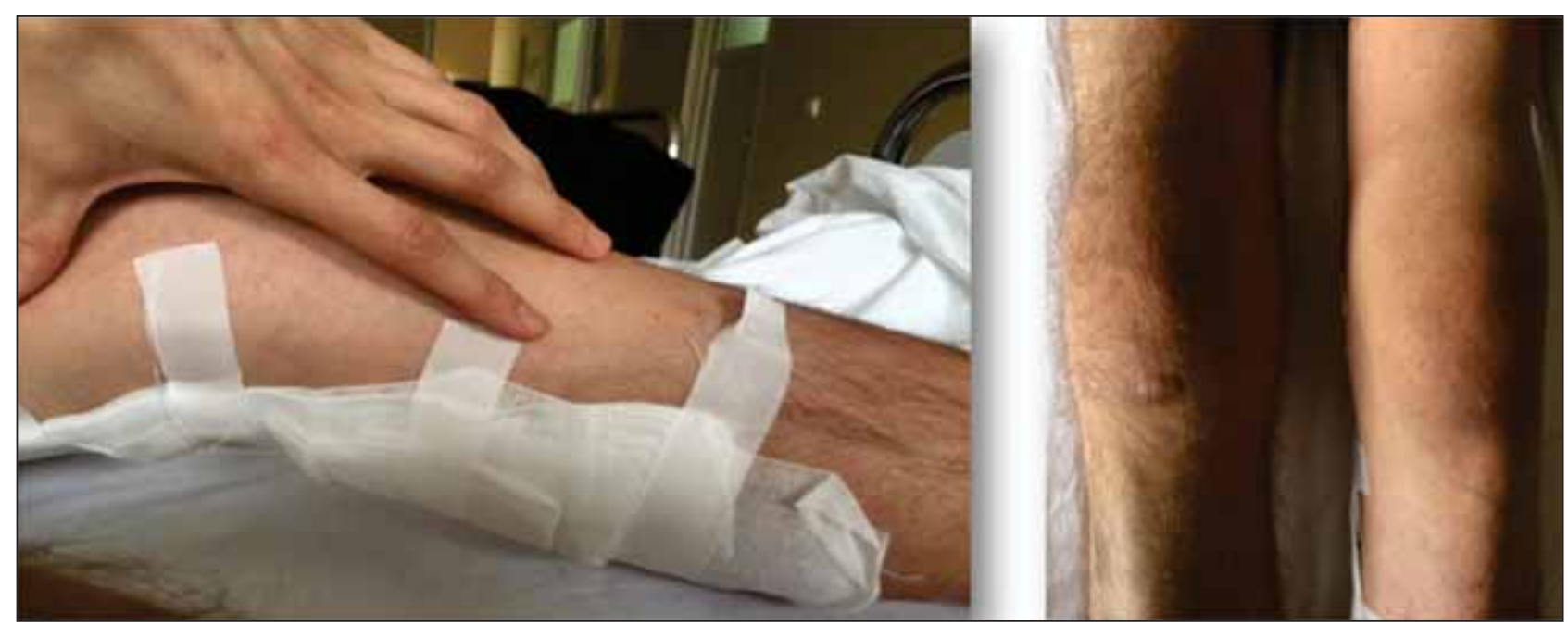

FIGURE 5. Fused left Baker's cyst with surgical intervention Source: Author's contribution

Concerning treatment, he is on temporary antipyretics, antibiotics (iv cephalosporin) and antithrombotic prophylaxis following his knee intervention. His long term management requires background drug therapy with Colchicine in a slow increase in dose according to patient's tolerability, Sulfasalazine and Methylprednisolone with an attempt to lower dose (4 mg qd). Patient also requires Teriparatide, calcium and vitamin D supplements for his severe osteoporosis. Reintroduction of biological therapy will be made possible after full recovery from his surgical intervention. Future actions demand surgical intervention for hip arthroplasty because of his osteonecrosis.

As highlights of this case we would bring into attention the unusual geographical area of FMF incidence and the fact that the patient reports no family history of FMF or other rheumatic conditions. The fact that the patient tested HLA B27 positive might not be surprising knowing that up to $47 \%$ in FMF patients can have this genetic background (6). Moreover, the long interval to correct diagnosis together with intense and longstanding corticosteroid therapy led to major consequences as previously mentioned. Patient being partially responsive to colchicine led to the need to initiate a TNF inhibitor because of the inaccessibility to an anti-ILI agent.

\section{DISCUSSIONS}

Familial Mediterranean Fever is the most frequent hereditary condition in the autoimmune diseases' group, having a specific ethnical distribution, occurring with a higher rate among Turks, Arabs and Jews (7). Its prevalence varies widely depending on the geographical setting and it is estimated at around $1 / 200-1 / 1,000$ patients (8). 


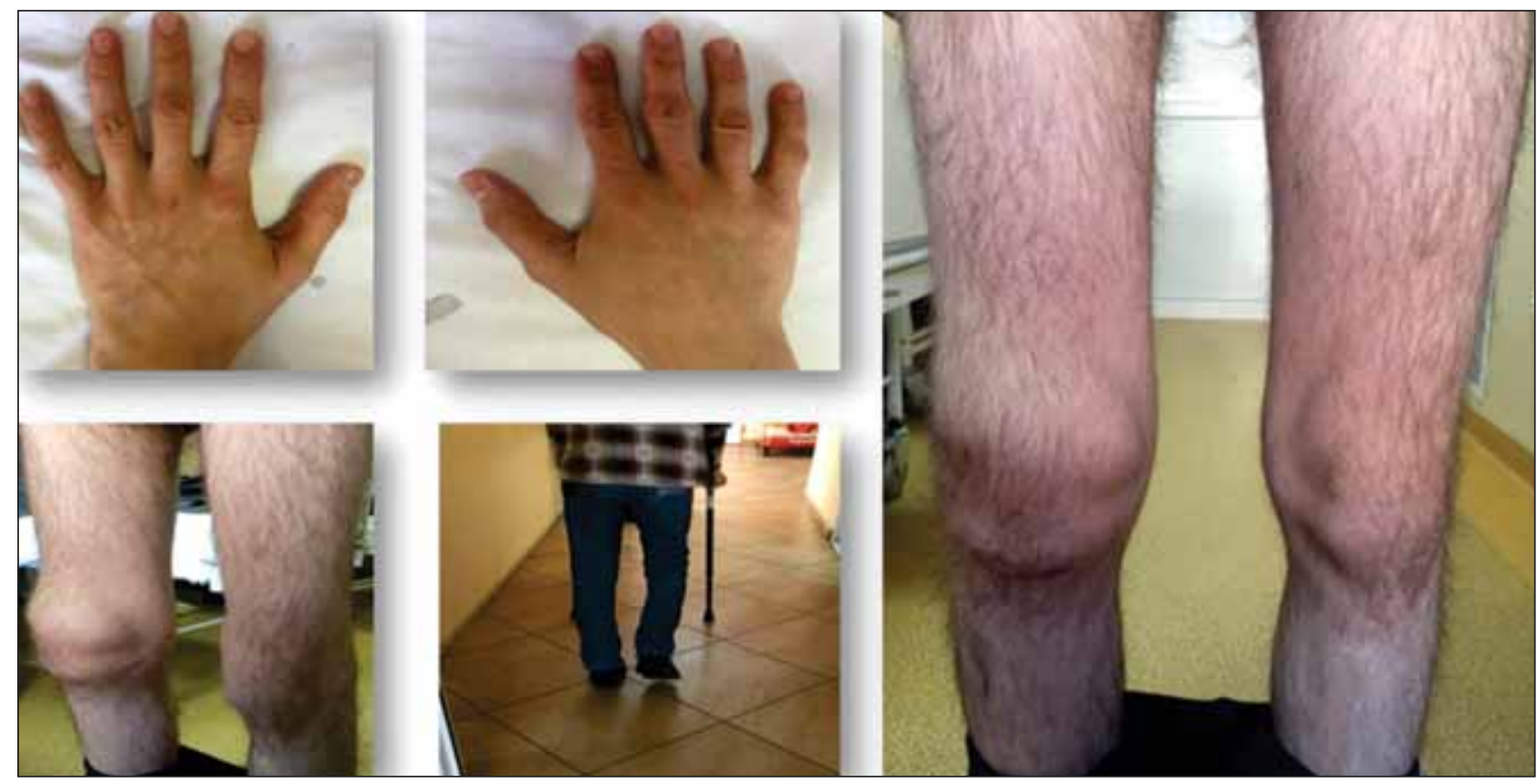

FIGURE 6. Patient's current state (no hand deformities, refractory knee arthritis)

Source: Author's contribution

FMF is an autosomal recessive transmitted disease affecting patients before the age of 30 (9) and the causative gene was proved to be the Mediterranean Fever gene (MEFV) located on the short arm of chromosome 16 comprising of 10 exons (10). The type of MEFV mutations may differ between racial subpopulations, but the most commonly seen is M694V (11).

The diagnostic suspicion of FMF is mainly based on clinical manifestations with recurrent febrile attacks, peritonitis, pericarditis or pleural effusion or arthritis affecting large joints (12).

Flares are short in duration (1-3 days) with remittance even in the absence of treatment and they can be triggered by stress, viral infections or physical activity (13).

One of the most common complaints is abdominal pain that can be either diffuse or localized, followed by joint involvement affecting mostly joints in the lower limbs. Swelling of the scrotum can occur but mostly in younger children (14). Other signs and symptoms include myalgia, splenomegaly, enlarged lymph nodes and various cutaneous findings (erysipelas-like erythema, urticaria, nodules) $(3,15)$.

Patients with FMF can also exhibit spondylitis or sacroiliitis, with an estimated rate of 7\% (3). Some authors suggest that patients with both spinal ankylosis and positive HLA B27 have two coinciding diseases - ankylosing spondylitis (AS) and FMF (16). This association brings up more interest, knowing that some literature publications state that the MEFV mutation is increased in AS patients (17).

The most fearful long-term complication of FMF is the development of AA-amyloidosis because of the sustainedly increased production of SAA protein, with further progression to renal disease that darkens patients' prognosis $(18,19)$. This manifests with proteinuria accompanied or not by hematuria which might require renal biopsy to confirm the diagnosis (20). Amyloid deposits might also be found in the spleen, the adrenal glands or the gastro-intestinal tract. The mean interval from disease onset to amyloidosis diagnosis is approximately 17 years (21).

The risk factors for developing FMF-related amyloidosis, as identified by Kaşifoglu et al. are male gender, M694V homozygotes or a positive family history of amyloidosis (22).

Diagnosis is based on the Tel-Hashomer criteria, having a satisfactory sensitivity and sensibility diagnostic rate (3).

A typical attack is defined by as recurrent (more than three episodes), febrile over $38^{\circ} \mathrm{C}$ and short (12 hours to three days), while incomplete attacks have a lower temperature, a shorter duration, there are no detectable signs of peritonitis and patients have arthritis in atypical joints $(24,25)$.

Current laboratory tests are non-specific for diagnosis of FMF; acute phase reactants such as ESR, $\mathrm{CRP}$, fibrinogen and serum amyloid A are increased during disease attacks (26). 


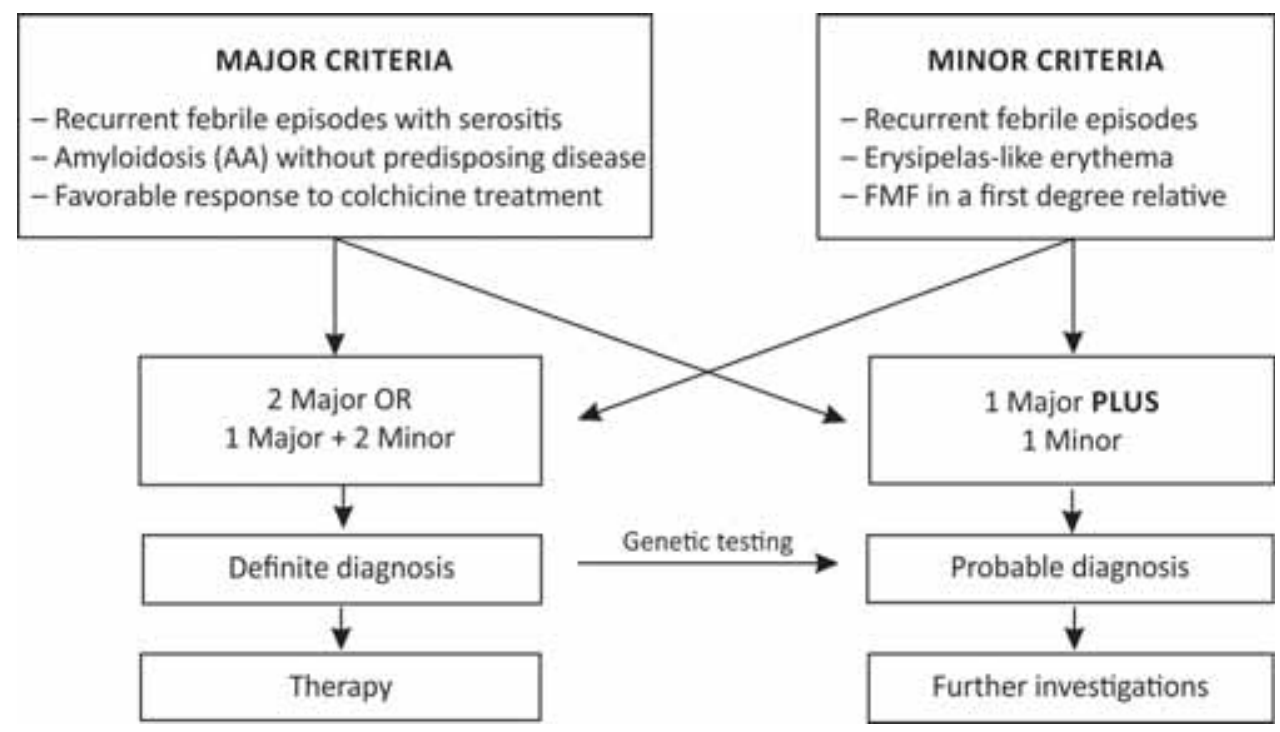

FIGURE 7. Tel-Hashomer diagnostic criteria for FMF Source: Adapted from Wang et al. (23)

Once the diagnosis is set, preferably by a physician with experience in FMF, treatment should aim to control disease attacks and to lower the subclinical inflammatory state.

According to the latest EULAR recommendations for the management of FMF, colchicine treatment should start as soon as the clinical diagnosis is made, as this represents the mainstay treatment in FMF (5). Adjusting dose is made according to patient tolerance and can be increased in periods of physical or emotional triggers. However, treatment toxicity, including liver enzymes and renal function, should be carefully monitored (27).

Although colchicine represents the "gold standard" treatment in FMF, around $10 \%$ of patients are non-responders or resistant (28). This category might benefit from biological therapy with IL-1 inhibitors (anakinra, canakinumab, rilonacept) or antiTNF agents such as etanercept, infliximab or adalimumab as seen in observational studies $(29,30)$.
Series of case reports also suggested the use of IL-6 blockade (tocilizumab) in suppressing the acute inflammatory response, proving its benefits especially in patients with amyloidosis secondary to FMF (31). Due to the relatively scarce data on the latter's efficacy, larger studies are expected in the near future.

\section{CONCLUSIONS}

This case presentation depicts the hardships of setting the right diagnosis in a case of late onset FMF due to unusual geographical setting, absence of suggestive family history and heterogeneous clinical presentation together with possibilities in therapeutic approach if patients are non or partially responsive to traditional therapies. Furthermore it points out possible drug side effects and comorbidities that require the same quality medical care as the main rheumatic disease.

\section{REFERENCES}

1. Livneh A., Langevitz P., Zemer D. et al. Criteria for the diagnosis of familial Mediterranean fever. Arthritis Rheum. 1997;40(10):18791885. doi:10.1002/1529-0131(199710)40:10\&lt;1879::AIDART23\&gt;3.0.CO;2-M.

2. Yalcinkaya F., Ozen S., Ozcakar Z.B. et al. A new set of criteria for the diagnosis of familial Mediterranean fever in childhood. Rheumatology. 2009; 48(4):395-398. doi:10.1093/rheumatology/ ken509.

3. Sari I., Birlik M., Kasifoglu T. Familial Mediterranean fever: An updated review. Eur J Rheumatol. 2014; 1(1):21-33. doi:10.5152/ eurjrheum.2014.006.

4. Dogan H., Akdemir F., Tasdemir S. et al. A novel insertion mutation identified in exon 10 of the MEFV gene associated with Familial Mediterranean Fever. BMC Med Genet. 2014; 15:74. doi:10.1186/1471-2350-15-74.

5. Ozen S., Demirkaya E., Erer B. et al. EULAR recommendations for the management of familial Mediterranean fever. Ann Rheum Dis. 2016; 75(4):644-651. doi:10.1136/annrheumdis-2015-208690.

6. Kaşifolu T., Çalişir C., Cansu D.Ü., Korkmaz C. The frequency of sacroiliitis in familial Mediterranean fever and the role of HLA-B27 and MEFV mutations in the development of sacroiliitis. Clin Rheumatol. 2009; 28(1):41-46. doi:10.1007/s10067-008-0980-3. 
7. Shohat M., Halpern G.J. Familial Mediterranean fever A review. Genet Med. 2011; 13(6):487-498. doi:10.1097/ GIM.0b013e3182060456.

8. Fujikura K. Global epidemiology of Familial Mediterranean fever mutations using population exome sequences. Mol Genet Genomic Med. 2015; 3(4):272-282. doi:10.1002/mgg3.140.

9. Kucuk A., Gezer I.A., Ucar R., Karahan A.Y. Familial Mediterranean Fever. Acta medica (Hradec Králové) / Univ Carolina, Fac Medica Hradec Králové. 2014; 57(3):97-104. doi:10.14712/18059694.2014.47.

10. Touitou I. The spectrum of Familial Mediterranean Fever (FMF) mutations. Eur J Hum Genet. 2001; 9(7):473-483. doi:10.1038/ sj.ejhg.5200658

11. Bernot A., da Silva C., Petit J.L. et al. Non-founder mutations in the MEFV gene establish this gene as the cause of familial Mediterranean fever (FMF). Hum Mol Genet. 1998; 7(8):1317-1325. doi:9668175.

12. Savic S., Dickie L.J., Battellino M., McDermott M.F. Familial Mediterranean fever and related periodic fever syndromes/ autoinflammatory diseases. Curr Opin Rheumatol. 2012; 24(1):103-112. doi:10.1097/BOR.0b013e32834dd2d5.

13. Ozen S., Bilginer Y. A clinical guide to autoinflammatory diseases: familial Mediterranean fever and next-of-kin. Nat Rev Rheumatol. 2014; 10(3):135-147. doi:10.1038/nrrheum.2013.174.

14. Livneh A., Langevitz P. Diagnostic and treatment concerns in familial Mediterranean fever. Baillieres Best Pract Res Clin Rheumatol. 2000; 14(3):477-498. doi:10.1053/berh.2000.0089.

15. Lidar M., Livneh A. Familial mediterranean fever: Clinical, molecular and management advancements. Neth J Med. 2007; 65(9):318-324. doi:10.1155/2013/185317.

16. Cosan F., Ãstek D., Oku B. et al. Association of familial Mediterranean fever-related MEFV variations with ankylosing spondylitis. Arthritis Rheum. 2010; 62(11):3232-3236. doi:10.1002/ art.27683.

17. Sarikaya S., Ozdolap S., Marasli E. Spondylitis and arthritis in familial mediterranean fever. Turkish J Rheumatol. 2012; 27(4):241-247. doi:10.5606/tjr.2012.043.

18. Hama I., Ilham R., Ouzeddoun N., Alhamany Z., Bayahia R., Sefiani A. Renal amyloidosis due to familial Mediterranean fever misdiagnosed. Indian J Hum Genet. 2012; 18(3):363. doi:10.4103/0971-6866.108043.

19. Ludomirsky A., Passwell J., Boichis H. Amyloidosis in children with familial Mediterranean fever. Arch Dis Child. 1981; 56(6):464467. doi:10.1136/adc.56.6.464.
20. Ebrahimi-Fakhari D., Schönland S.O., Hegenbart U. et al. Familial Mediterranean fever in Germany: clinical presentation and amyloidosis risk. Scand J Rheumatol. 2013; 42(1):52-58. doi:10.31 09/03009742.2012.714796.

21. Medlej-Hashim M., Delague V., Chouery E. et al. Amyloidosis in familial Mediterranean fever patients: correlation with MEFV genotype and SAA1 and MICA polymorphisms effects. BMC Med Genet. 2004; 5:4. doi:10.1186/1471-2350-5-4.

22. Kasifoglu T., Bilge S.Y., Sari I. et al. Amyloidosis and its related factors in Turkish patients with familial Mediterranean fever: a multicentre study. Rheumatology (Oxford). 2014; 53(December 2013):741-745. doi:10.1093/rheumatology/ket400.

23. Bonfrat L., Bonfrate L., Bari $O$ de, Wang T.Y., Portincasa P. Familial Mediterranean Fever: From Pathogenesis to Treatment. J Genet Syndr Gene Ther. 2014; 05(05):1-11. doi:10.4172/2157-7412.1000248.

24. Onen F. Familial Mediterranean fever. Rheumatol Int. 2006; 26(6):489-496. doi:10.1007/s00296-005-0074-3.

25. Berkun Y., Eisenstein E.M. Diagnostic criteria of familial Mediterranean fever. Autoimmun Rev. 2014; 13(4-5):388-390. doi:10.1016/j.autrev.2014.01.045.

26. Zadeh N., Getzug T., Grody W.W. Diagnosis and management of familial Mediterranean fever: Integrating medical genetics in a dedicated interdisciplinary clinic. Genet Med. 2011; 13(3):263-269. doi:10.1097/GIM.0b013e31820e27b1.

27. Zemer D., Livneh A., Danon Y.L., Pras M., Sohar E. Long-term colchicine treatment in children with familial mediterranean fever. Arthritis Rheum. 1991; 34(8):973-977. doi:10.1002/art.1780340806.

28. Koga T., Migita K., Kawakami A. Biologic therapy in familial Mediterranean fever. Mod Rheumatol. 2016; 26(5):637-641. doi:10. 3109/14397595.2016.1162261.

29. Ozgur A., Kilic E., Kilic G., Ozgocmen S. Efficacy and Safety of Biologic Treatments in Familial Mediterranean Fever. Am J Med Sci. 2013; 346(2):137-141. doi:10.1097/MAJ.0b013e318277083b.

30. Van der Hilst J.C., Moutschen M., Messiaen P.E., Lauwerys B.R., Vanderschueren S. Efficacy of anti-IL-1 treatment in familial Mediterranean fever: a systematic review of the literature. Biologics. 2016; 10:75-80. doi:10.2147/BTT.S102954.

31. Umeda M., Aramaki T., Fujikawa K. et al. Tocilizumab is effective in a familial Mediterranean fever patient complicated with histologically proven recurrent fasciitis and myositis. Int $J$ Rheum Dis. October 2015:n/a - n/a. doi:10.1111/1756-185X.12776.

Conflict of interest: none declared Financial support: none declared 